\title{
How to dose infliximab in rheumatoid arthritis: new data on a serious issue
}

\section{Ronald F van Vollenhoven}

\author{
"Never go to excess, but let moderation \\ be your guide" Cicero \\ "Too much of a good thing is wonder- \\ ful" Mae West
}

The approval of the monoclonal antitumour necrosis factor antibody, infliximab, for the treatment of rheumatoid arthritis (RA) in 1999, occurring almost simultaneously with that of etanercept, marked an important event in the history of rheumatology. The possibility of providing good disease control to patients with the disease, while preventing structural damage, increased dramatically, and rheumatology would never be the same.

From the time of its first approval, a peculiar feature of infliximab was uncertainty about optimal dosing. The original observations made at the Kennedy Institute in London with the antibody then known as cA2, later as "avakine", and later still as infliximab, suggested that $1 \mathrm{mg} / \mathrm{kg}$ was too little to provide meaningful responses. On the other hand, $3 \mathrm{mg} / \mathrm{kg}, 10 \mathrm{mg} / \mathrm{kg}$ and even $20 \mathrm{mg} / \mathrm{kg}$ were found to have beneficial effects in RA. ${ }^{1-4}$ The single pivotal phase III clinical trial carried out with infliximab, the ATTRACT study, tested four possible combinations of dosage and dosing interval: $3 \mathrm{mg} / \mathrm{kg}$ or $10 \mathrm{mg} / \mathrm{kg}$, every 4 or every 8 weeks. ${ }^{4}$ It should perhaps be noted that in that trial each of these dosage/ frequency combinations was preceded by the-now standard-_loading" regimen of three infliximab infusions given over 6 weeks (at 0,2 , and 6 weeks). There is, to the best of my knowledge, no clear pharmacological rationale for such a loading dose, but it certainly has made it more difficult accurately to assess and interpret the results with the various dosage/ frequency combinations. For example, the 24-week results in the ATTRACT trial were very similar for the four different infliximab dosage/frequency groups (and, of course, much better than placebo); but was this evidence that each

Correspondence to: Ronald F van Vollenhoven, The Karolinska Institute, Stockholm 17176, Sweden; ronald. van.vollenhoven@ki.se of these options was equal, or was it simply the carry-over effect of an initial period with a higher level of infliximab treatment? A slight decrease in American College of Rheumatology responses for the groups receiving the lower dosages at 48 weeks suggested the latter possibility; and authorities in the USA and Europe, employing slightly different ways of dealing with the dosage/frequency problem, ended up issuing approvals that gave clinicians in many countries significant leeway in choosing dosages and frequencies for individual patients.

Matters were not made much easier when a subsequent large trial of infliximab in early RA was published showing that $6 \mathrm{mg} / \mathrm{kg}$ was slightly, but not significantly, more effective than $3 \mathrm{mg} / \mathrm{kg}$. ${ }^{6}$ Nor was it helpful that, parallel to these developments in RA, infliximab was approved for use in ankylosing spondylitis and some other indications at the $5 \mathrm{mg} / \mathrm{kg}$ dose.

At the same time, various reports from uncontrolled, observational studies suggested that patients treated with the lowest approved dosage of $3 \mathrm{mg} / \mathrm{kg}$ every 8 weeks sometimes displayed a good initial response but then seemed to do worse after settling down on that dose (ie, after the effect of the initial "loading" subsided), and that this "secondary loss of efficacy" could then be effectively recaptured with higher dosages. ${ }^{7}$ The same modest decrease in overall efficacy with standard-dose infliximab was also noted in a randomised trial featuring both abatacept and infliximab.

As an important point on the pharmacodynamics of infliximab, St Clair et al showed that in order to achieve adequate trough levels of infliximab the more effective strategy is to reduce the dosing interval rather than to increase the dosage. ${ }^{9}$ For example, $3 \mathrm{mg} / \mathrm{kg}$ every 6 weeks resulted in higher trough levels than $4-5 \mathrm{mg} / \mathrm{kg}$ every 8 weeks, and under the assumption that trough levels are what matters for efficacy, the higher frequency would give you a "better bang for your buck".

Among several uncontrolled observational studies dealing with the problem of infliximab dosing in RA, our own study from 2004 reported on patients whose infliximab dose was increased in actual practice. $^{10}$ These patients appeared to benefit from the dosage increase-that is, their 28-joint count Disease Activity Score (DAS28) values after the dosage increase were better than before. However, we considered the possibility that this improvement might represent "regression to the mean". This is the statistical phenomenon that a measurement made in a group of people who are selected from a larger group based on having values at one or the other end of the range will tend to return to levels closer to the mean for the larger group. To put it more simply, if you are doing poorly at any given point in time, chances are you will start improving again. And indeed, when we compared our patients whose dosages had been increased with matched patients whose dosage had not been increased, the results were exactly the same (fig 1).

Another important observational result was recently published in the Annals by van den Bemt et al ${ }^{11}$ : in a group of 18 patients who were being treated with infliximab at doses $>3 \mathrm{mg} / \mathrm{kg}$, reduction of the dose to $3 \mathrm{mg} / \mathrm{kg}$ did not result in a flare of the disease in 17 of those 18 patients - which would have been a plausible result if no change had been made at all.

Based on these results, mostly from observational studies, serious clinicians might well have started wondering what exactly they were achieving by prescribing higher dosages of infliximab to their patients with RA.

After this much uncertainty, it was heartening to note that a randomised controlled trial had been performed dealing with this very question. In this issue of the Annals, Pavelka et a ${ }^{12}$ report a study on patients with RA who were initially treated with infliximab at $3 \mathrm{mg} / \mathrm{kg}$ and included in the Czech ATTRA biologics registry (see article on page 1285). Patients who did respond to the treatment (DAS28 improved by at least 1.2) but who failed to achieve remission (DAS28 >2.6) were eligible for the randomised trial, and 140 patients were included. These patients were randomised either to continue infliximab at the same dosage (group A) or to increase the dosage to $5 \mathrm{mg} / \mathrm{kg}$ (group B), both doses given every 8 weeks. The primary end point of the study was the change in DAS28 assessed 28 weeks after randomisation. It was found that in group $\mathrm{A}$, the 71 patients whose dosage was unchanged, the mean DAS28 improved from 4.5 to 4.0, and in group B, the 69 patients whose dosage was 


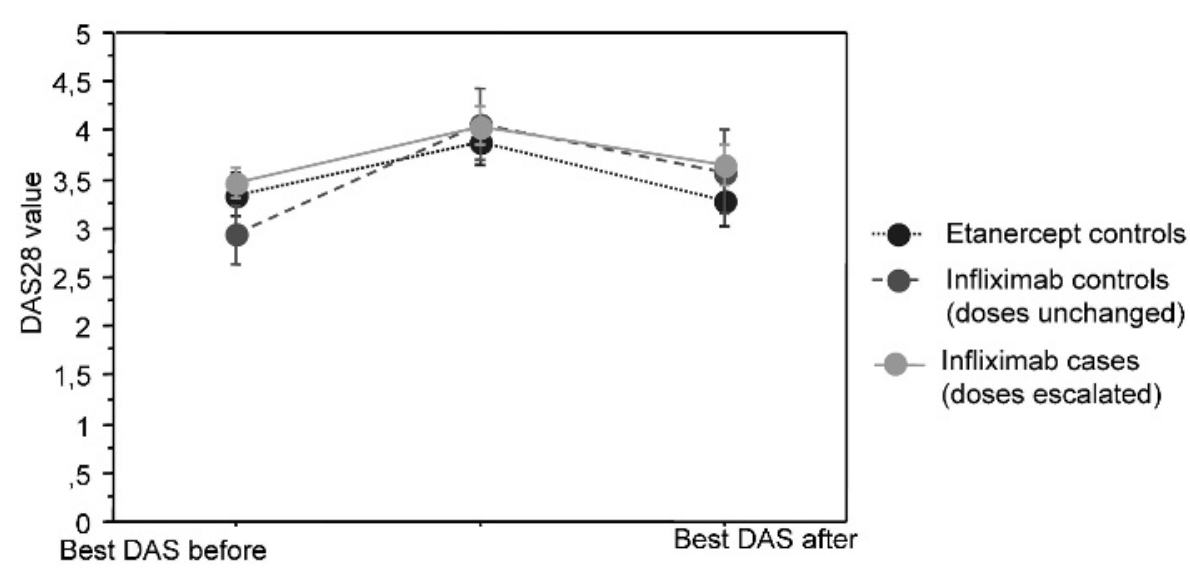

Figure 1 Three groups of patients from the Stockholm registry were compared: patients with rheumatoid arthritis whose infliximab dosage was increased (infliximab "cases"), patients whose dosage was not increased (infliximab "controls") and patients receiving a stable dose of etanercept (etanercept controls). For the cases, the values are: best 28 -joint count Disease Activity Score (DAS28) before dose escalation, DAS28 at dose escalation and best DAS28 after dose escalation. For the two control groups, the values are: best DAS28 during treatment, first DAS28 after that to show an increase and best DAS28 after that time point. As can clearly be seen, the improvement seen after dose increase is mirrored by improvements in the control groups, suggesting these improvements are due to regression to the mean rather than an actual therapeutic effect. (Reproduced, with permission, from Van Vollenhoven et al. ${ }^{10}$ )

increased, the DAS28 improved from 4.5 to 3.9. Clearly, there was no meaningful difference between these groups, and the same held true for all secondary outcomes studied. A statistically significant increase in the incidence of adverse events was noted in the higher-dose group. The authors conclude that the dose increase did not improve efficacy.

This was a well-designed clinical trial that provided a clear answer to an important question. As is the case for all studies, some weaknesses can be noted, but none that would, in my opinion, detract from the conclusions. The size of the trial allows for a small but true difference in efficacy to go unnoticed, but the magnitude of such a true difference would have to be so small as to render it clinically meaningless. Even higher doses of infliximab might have had added benefit had they been studied, but to posit that seems a real stretch. Radiological outcomes were not studied. The increase in adverse events with the higher dose is of concern, even if the frequencies of serious adverse events and serious infections were not higher.

An interesting point to note is that, while the average improvement in both groups was modest, there were undoubtedly patients in each group who improved substantially after enrolment. I have no doubt that those patients, and their doctors, feel quite certain that the dose increase really helped them a lot!

All in all, it would appear that we now have high-level evidence that increasing the infliximab dosage is of no benefit in RA, and any observational data supporting the contrary view will have to be held against the new standard set by Pavelka et al.

So what are clinicians to make of this? From informal conversations that I have had over the years, it is clear that most clinicians who use infliximab are deeply convinced that many of their patients need higher dosages than the recommended $3 \mathrm{mg} / \mathrm{kg}$ - and some of my own patients share in that conviction. This touches on a sensitive nerve: the observations made by serious, experienced doctors in clinical practice are, in today's over-regulated climate, all too easily dismissed in favour of "evidence-based guidelines" and the like, which attempt to apply results obtained under highly controlled circumstances at the group level to the unique individual. I would be the last person to suggest diminishing the doctor's authority in therapeutic decision-making. But nonetheless: the capacity of the human mind to be fooled by appearances is breathtaking (fig 2). Is it possible that so many of us have been fooled so many times? Perhaps it is. Undoubtedly, in the study by van den Bemt described above, the doctors who had prescribed the higher dosage of infliximab were entirely convinced that it was necessary, yet they were demonstrably wrong.

Box 1 presents a modest proposal for the application of these results to clinical practice. The initial dosage of infliximab must be $3 \mathrm{mg} / \mathrm{kg}$ given every 8 weeks.
Patients who experience a clear "break" in the clinical effect that occurs 4-7 weeks after each infusion can be treated at the interval suggested by that report. In patients who achieve partial improvement with infliximab but for whom disease control is not considered adequate, a dose increase should not be chosen; other drugs can be tried. However, in contrast to the approach chosen by Pavelka et al, it may be too ambitious to try to achieve remission in every patient with established RA; a more realistic treatment goal might be low disease activity. And in patients who are already receiving a greater dose of infliximab, an attempt should be made to reduce the dosage to $3 \mathrm{mg} / \mathrm{kg}$.

In recent years, several registry studies have suggested that a sizeable proportion of patients with RA are receiving infliximab at dosages $>3 \mathrm{mg} / \mathrm{kg}$. Thus, in the Swiss registry, after 2 years around $20 \%$ of patients were being treated with a higher dosage. ${ }^{13}$ In our own STURE registry in the Stockholm region, after 4 years $45 \%$ of patients were receiving a higher dose. ${ }^{14}$ Over the entire treatment duration of RA with infliximab, which in our study was a median of 6 years, this resulted in an average overdosing of $30 \%$. In the larger European context, German, French, Italian and Spanish rheumatologists have had considerable freedom in applying higher dosages, and it is reasonable to assume similar proportions of patients are receiving higher dosages as in the two smaller countries. (The UK, on the other hand, has had more restrictive reimbursement policies, and in many of the world's countries cost has been a limiting factor.)

Total sales of Remicade during the past 10 years can be estimated at $€ 10$ billion. ${ }^{14}$ If we make the following assumptions: that the use of Remicade in rheumatology represents $80 \%$ of the total; that $70 \%$ of Remicade use in rheumatology is for RA; that dosing of Remicade in RA is not limited to $3 \mathrm{mg} / \mathrm{kg}$ by regulators or reimbursers in $75 \%$ of the "market"; and, as indicated above, that Remicade in RA overdosing averages out to 30/130 or $23 \%$ of the total, then it can be quickly calculated that about $€ 1$ billion has been spent unnecessarily. In this age of multibillion dollar bailouts for incompetent bankers I don't wish to overplay the mere numbers, but in this case, an important lesson is to be learnt, and it is this: We, as rheumatologists in practice and in academia, must take on the responsibility for determining the optimal use of antirheumatic drugs. We must take this responsibility seriously, using all available resources for carrying out high-quality 


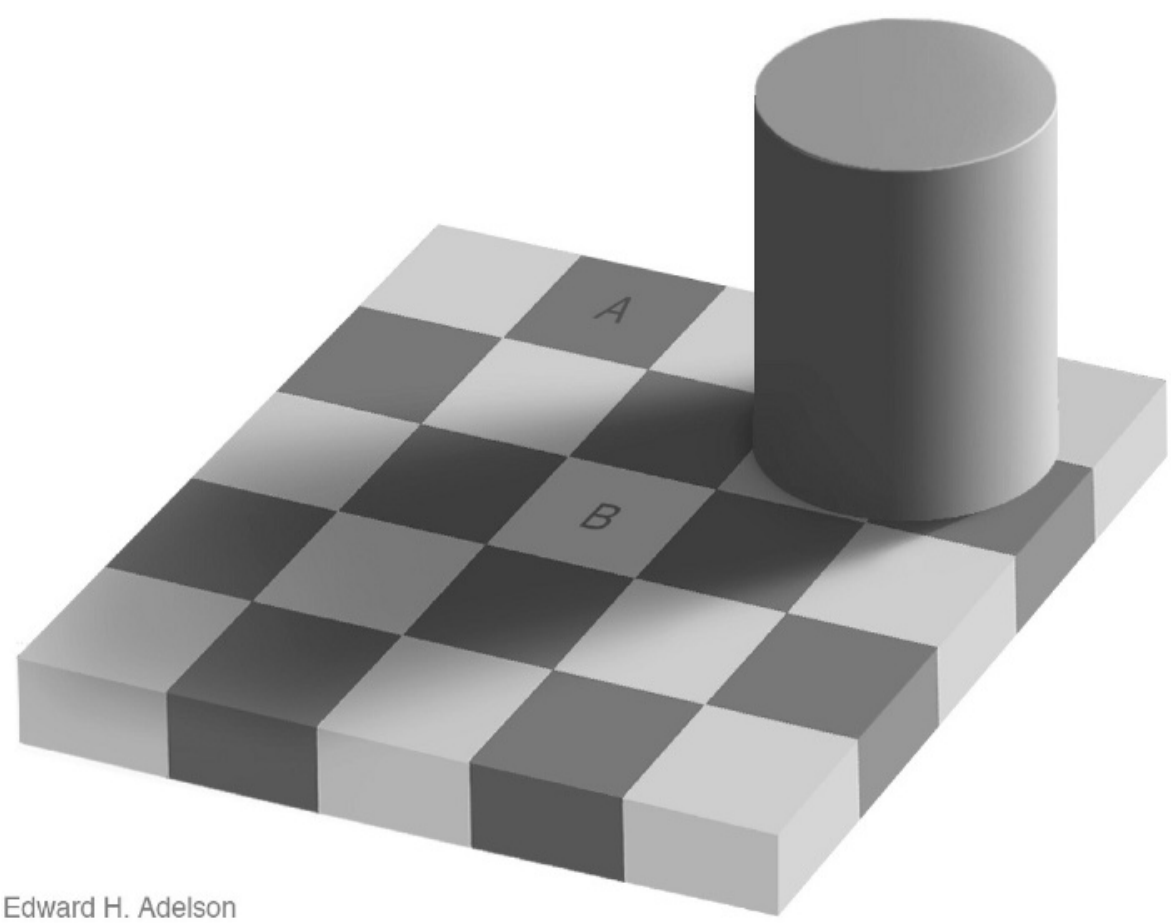

Figure 2 A powerful optical illusion: The squares marked A and B are the same tone of grey! Illusion created by Edward H Adelson, permission for use granted on http://web.mit.edu/persci/ people/adelson/checkershadow_illusion.html (accessed 4 June 2009).

studies and trials; and if these give us a result that is clear, we must implement it and monitor the outcome. Each time we fail to do so, it will provide added fuel to those who are insisting on regulating the prescription of drugs at levels far above the individual doctor, to the detriment of our profession.

Thus, there is a feeling of sadness when it turns out that it is most likely that a very large number of patients have been treated for many years with dosages of infliximab that were unnecessarily high. On the other hand, doctors have to work with incomplete data and overtreating may be a lesser evil than undertreating. The most encouraging news today is that Pavelka et al have demonstrated how serious academic rheumatologists working jointly with clinicians can answer the questions that industry and regulators will not, enabling us to treat our future patients even better than we have treated our previous ones.

\section{Competing interests: None.}

Ann Rheum Dis 2009;68:1237-1239.

doi:10.1136/ard.2009.111682

\section{Box 1 Recommendations for infliximab dosing in rheumatoid arthritis}

- The initial dosage should be $3 \mathrm{mg} / \mathrm{kg}$ for each infusion

- note that the value of "loading" (an infusion given at weeks 0, 2 and 6) has never been formally proved

- The usual frequency is every 8 weeks

- A convincing patient history that the benefit experienced after each infusion lasts for $<8$ full weeks could lead to a reduction of the infusion interval to that indicated by the history

- There is no role for dosage increases

- If the benefit of treatment is inadequate (lack of response, or partial response), other treatments should be considered

- For patients who are already receiving infliximab at a higher dosage, an attempt should be made to reduce it to $3 \mathrm{mg} / \mathrm{kg}$

\section{REFERENCES}

1. Elliott MJ, Maini RN, Feldmann M, Long-Fox A, Charles P, Bijl H, et al. Repeated therapy with monoclonal antibody to tumour necrosis factor alpha (cA2) in patients with rheumatoid arthritis. Lancet 1994;344:1125-7.

2. Elliott MJ, Maini RN, Feldmann M, Long-Fox A, Charles $\mathrm{P}$, Katsikis $\mathrm{P}$, et al. Treatment of rheumatoid arthritis with chimeric monoclonal antibodies to tumor necrosis factor alpha. Arthritis rheum 1993;36:1681-90.

3. Elliott MJ, Maini RN, Feldmann M, Kalden JR, Antoni C, Smolen JS, et al. Randomised double-blind comparison of chimeric monoclonal antibody to tumour necrosis factor alpha (cA2) versus placebo in rheumatoid arthritis. Lancet 1994;344:1105-10.

4. Maini R, St Clair EW, Breedveld F, Furst D, Kalden J, Weisman M, et al. Infliximab (chimeric anti-tumour necrosis factor alpha monoclonal antibody) versus placebo in rheumatoid arthritis patients receiving concomitant methotrexate: a randomised phase III trial. ATTRACT Study Group. Lancet 1999:354:1932-9.

5. Lipsky PE, van der Heijde DM, St Clair EW, Furst DE, Breedveld FC, Kalden JR, et al. Infliximab and methotrexate in the treatment of rheumatoid arthritis. Anti-Tumor Necrosis Factor Trial in Rheumatoid Arthritis with Concomitant Therapy Study Group. N Engl J Med 2000;343:1594-602.

6. St Clair EW, van der Heijde DM, Smolen JS, Maini RN, Bathon JM, Emery P, et al. Combination of infliximab and methotrexate therapy for early rheumatoid arthritis: a randomized, controlled trial. Arthritis Rheum 2004:50:3432-43.

7. Durez P, Van den Bosch F, Corluy L, Veys EM, De Clerck L, Peretz A, et al. A dose adjustment in patients with rheumatoid arthritis not optimally responding to a standard dose of infliximab of $3 \mathrm{mg} /$ kg every 8 weeks can be effective: a Belgian prospective study. Rheumatology (Oxford) 2005;44:465-8

8. Schiff M, Keiserman M, Codding C, Songcharoen S Berman A, Nayiager $S$, et al. Efficacy and safety of abatacept or infliximab vs placebo in ATTEST: a phase III, multi-centre, randomised, double-blind, placebocontrolled study in patients with rheumatoid arthritis and an inadequate response to methotrexate. Ann Rheum Dis 2008;67:1096-103.

9. St Clair EW, Wagner CL, Fasanmade AA, Wang B Schaible T, Kavanaugh A, et al. The relationship of serum infliximab concentrations to clinical improvement in rheumatoid arthritis: results from ATTRACT, a multicenter, randomized, double-blind, placebo-controlled trial. Arthritis Rheum 2002;46:1451-9.

10. van Vollenhoven RF, Brannemark S, Klareskog L. Dose escalation of infliximab in clinical practice: improvements seen may be explained by a regression-like effect. Ann Rheum Dis 2004;63:426-30

11. van den Bemt BJ, den Broeder $A A$, Snijders GF, Hekster YA, van Riel PL, Benraad B, et al. Sustained effect after lowering high-dose infliximab in patients with rheumatoid arthritis: a prospective dose titration study. Ann Rheum Dis 2008;67:1697-701.

12. Pavelka K, Jarosova K, Suchy D, Senolt L, Chroust K, Dusek $L$, et al. Increasing the infliximab dose in rheumatoid arthritis patients: a randomised, doubleblind study failed to confirm its efficacy. Ann Rheum Dis 2009;68:1285-9.

13. Finckh A, Simard JF, Gabay C, Guerne PA. Evidence for differential acquired drug resistance to anti-tumour necrosis factor agents in rheumatoid arthritis. Ann Rheum Dis 2006:65:746-52.

14. van Vollenhoven RF, Klareskog L. Infliximab dosage and infusion frequency in clinical practice: experiences in the Stockholm biologics registry STURE. Scand J Rheumatol 2007;36:418-23. 